\title{
Contribution of diffusion weighted MRI to diagnosis and staging in gastric tumors and comparison with multi-detector computed tomography
}

\author{
Harun Arslan ${ }^{1}$, Mehmet Fatih Özbay², İskan Çall1³, Erkan Doğan4, Sebahattin Çelik ${ }^{5}$, \\ Abdussamet Batur ${ }^{1}$, Aydın Bora' ${ }^{1}$, Alpaslan Yavuz ${ }^{1}$, Mehmet Deniz Bulut ${ }^{1}$, Mesut Özgökçe ${ }^{1}$, \\ Mehmet Cetin Kotan ${ }^{5}$
}

\footnotetext{
1 YuzuncuYil University DursunOdabas Medical Center, Department of Radiology, Van, Turkey

2 Van Training and Research Hospital,Department of İnternal Medicine, Van, Turkey

${ }^{3}$ Van Training and Research Hospital,Department of General Surgery, Van, Turkey

${ }^{4}$ YuzuncuYil University DursunOdabas Medical Center, Department of Medical Oncology, Van, Turkey

${ }^{5}$ YuzuncuYil University DursunOdabas Medical Center, Department of General Surgery, Van, Turkey
}

Radiol Oncol 2017; 51(1): 23-29.

Received 7 February 2016

Accepted 24 August 2016

Correspondence to: HarunArslan, M.D., YuzuncuYil University, DursunOdabas Medical Center, Department of Radiology, Van, Turkey. Phone: +9 053274688 35; E-mail: harun.ars75@gmail.com

Disclosure: No potential conflicts of interest were disclosed.

Background. Diagnostic performance of Diffusion-Weighted magnetic resonance Imaging (DWI) and Multi-Detector Computed Tomography (MDCT) for TNM (Tumor, Lymph node, Metastasis) staging of gastric cancer was compared. Patients and methods. We used axial T2-weighted images and DWI (b-0,400 and b-800 s/mm²) protocol on 51 pre-operative patients who had been diagnosed with gastric cancer. We also conducted MDCT examinations on them. We looked for a signal increase in the series of DWI images. The depth of tumor invasion in the stomach wall (tumor (T) staging), the involvement of lymph nodes (nodal (N) staging), and the presence or absence of metastases (metastatic staging) in DWI and CT images according to the TNM staging system were evaluated. In each diagnosis of the tumors, sensitivity, specificity, positive and negative accuracy rates of DWI and MDCT examinations were found through a comparison with the results of the surgical pathology, which is the gold standard method. In addition to the compatibilities of each examination with surgical pathology, kappa statistics were used.

Results. Sensitivity and specificity of DWI and MDCT in lymph node staging were as follows: N1: DWI: $75.0 \%, 84.6 \%$; MDCT: 66.7\%, 82\%;N2: DWI: 79.3\%, 77.3\%; MDCT: 69.0\%, 68.2\%; N3: DWI: 60.0\%, 97.6\%; MDCT: 50.0\%, 90.2\%. The diagnostic tool DWI seemed more compatible with the gold standard method (surgical pathology), especially in the staging of lymph node, when compared to MDCT. On the other hand, in T staging, the results of DWI and MDCT were better than the gold standard when the T stage increased. However, DWI did not demonstrate superiority to MDCT. The sensitivity and specificity of both imaging techniques for detecting distant metastasis were $100 \%$.

Conclusions. The diagnostic accuracy of DWI for TNM staging in gastric cancer before surgery is at a comparable level with MDCT and adding DWI to routine protocol of evaluating lymph nodes metastasis might increase diagnostic accuracy.

Key words: gastric cancer; diffusion weighted imaging; MDCT; staging

\section{Introduction}

Although there are important geographical differences worldwide, death due to gastric cancer is the third leading cause of death from cancer. When clinically detected, it has already been in the advanced stage and made metastasis. ${ }^{1}$ Indistinct clinical presentation of gastric cancer is the main reason 


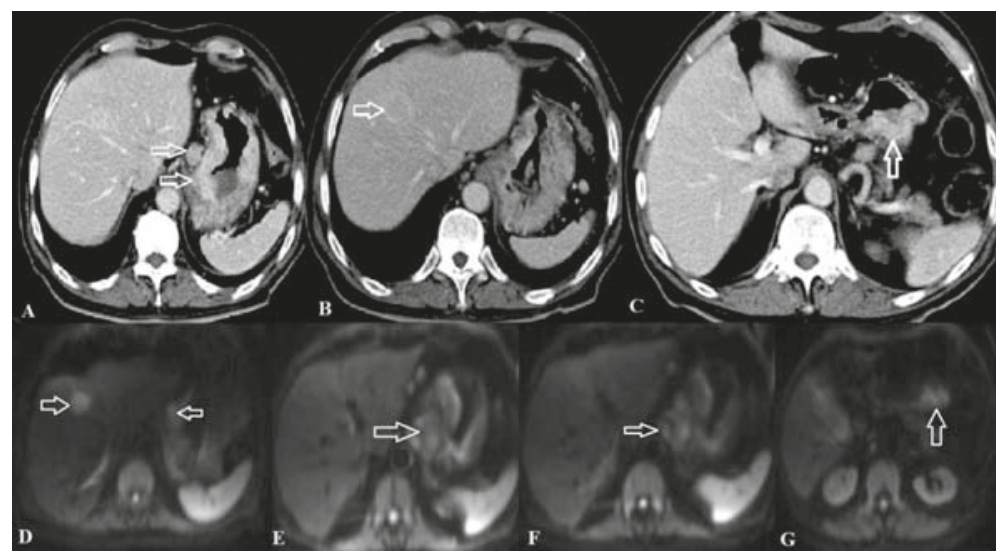

FIGURE 1. T4N3MI C Axial MDCT $(A, B, C)$ and $D W I(D, E, D, F, G)$. (A) Axial contrastedenhanced MDCT shows gastric corpus tumor and adjacent lymphadenopathies, (B) peripherally contrast enhanced lesions in the liver (metastasis), and (C) an invasive mass extending from the posterior of the gastric corpus to the fatty tissue (arrows). On DWI (D-G): on b800 DWI (D, E) there are diffusion restrictions in the gastric corpus and liver (Gastric tumor + liver metastasis), (F) restrictions that are compatible with lymphadenopathies are seen in the gastro-hepatic ligament, hepatic hilum, and the celiac axis, and (G) invasion of the fatty tissue in the posterior of the gastric corpus (arrow).

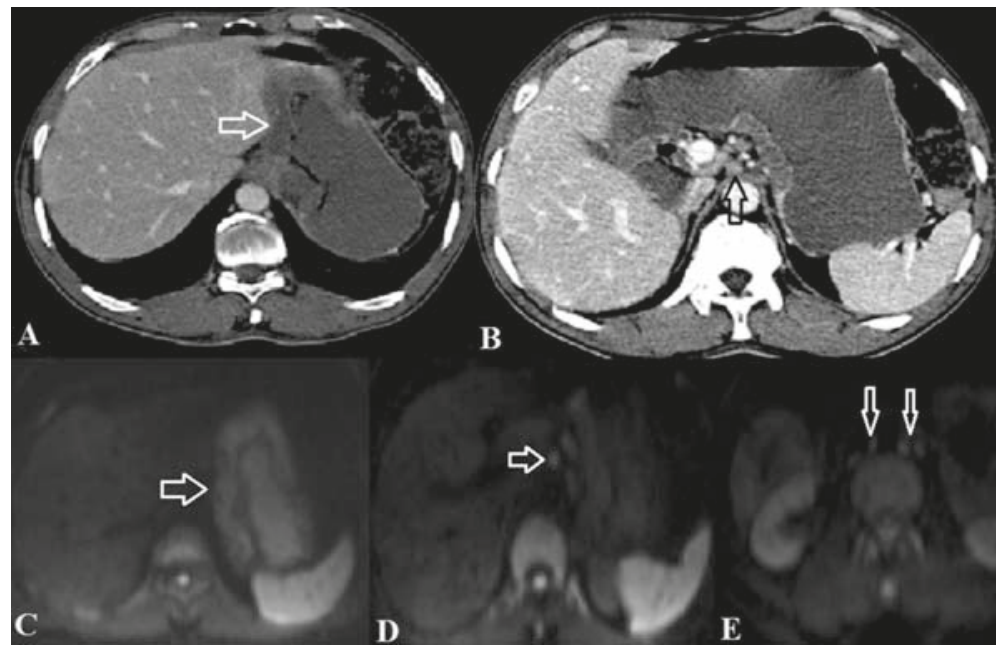

FIGURE 2. T2N3MO C Axial MDCT (A, B) and DWI (C,D,E). (A,B) axialcontrast-enhanced MDCT: Wall thickness in the gastric cardia-corpus and gastro-hepatic ligament and para-aorticlymphadenopathies, (C, D, E) b800 DWI images: (C) Diffusion restriction in the gastric cardia and corpus, (D) $\mathrm{mm}$ sized lymphadenopathiesin the gastrohepatic ligament, and(e)para-aorticandceliac lymphadenopathies.

for late diagnosis. Symptoms are generally nonspecific or all symptoms do not appear together. ${ }^{2}$ Recently, methods such as gastroscopy, endoscopic ultrasonography (EUS), computed tomography (CT), and positron emission tomography PET/CT are generally used for the staging of gastric cancer. ${ }^{3}$ With multi-dimensional reformatted (MPR) images and technical progress in multi-detector CT (MDCT) technology, which allows 3D imaging of the endoluminal surface of the stomach with thinsection axial images, MDCT is also generally used for the pre-operative evaluation of gastric cancer staging. ${ }^{4}$ However, in the clinical approach, some patients are not appropriate for contrast-enhanced CT due to renal dysfunction, hypersensitivity reaction to iodine-containing contrast agents, or they are avoiding radiation exposure because of the possibility of pregnancy. ${ }^{4,5}$ In patients with suspected gastric cancer and to whom contrasted CT cannot be applied, MRI has been mostly used for the diagnosis and staging of gastric cancer as an alternative imaging method to MDCT in the evaluation of gastric cancer.6,7 Especially, diffusion-weighted magnetic resonance imaging (DWI), whose main feature is to reflect movement of water molecules in tissue and gather information about tissue integration, was investigated in various tumors and the value of DWI was confirmed with various studies in detecting and characterizing gastric cancers. Furthermore, it can provide information for the differentiation of benign lesions from malignant ones, detecting malignant lymphadenopathy or very small peritoneal seeding and tissue cellularity, which might be used for monitoring therapeutic efficacy. DWI, draws attention as an oncologic imaging tool. ${ }^{8-10}$

In our study, we tested our hypothesis that DWI might be useful in staging based on pre-operative diagnostic efficacy of DWI and MDCT in gastric cancer patients and correlation analysis of surgical pathology.

\section{Patients and methods}

Between the periods of April 2013 and May 2015, fifty-one patients with malignant gastric adenocarcinoma diagnosis were included in the current study and their diagnosis was made with endoscopic biopsy in our hospital. In 51 gastric cancer patients, who were included in research and whose preoperative clinical TNM (Tumor-NodeMetastasis) staging were made, 37 were male and 14 were female. Their average age was 61 (range: 35 - 82) years old. Approval was taken from a local ethics committee and written consent was obtained from all patients.

First, diffusion-weighted magnetic resonance imaging (DWI) and upper and lower abdomen CT were taken pre-operatively from all patients. All examinations were made with multi-slice CT device (Siemens SOMATOM Sensation 16, Germany). In the $\mathrm{CT}, \mathrm{kV}$ was 120 and $\mathrm{mAs}$ was 150 . Each pa- 
tient was examined after eight hours of fasting. Stomach fullness was achieved by giving the patient one liter of water during the examination. By entering the right antecubital vein with an 18G needle, $100 \mathrm{ml}$ of contrast agent was administered (Omnipaque $350 \mathrm{mg}$, GE) at a rate of $2 \mathrm{ml} / \mathrm{s}$ by an automatic injector (Medrad, vision CT injection system). Approximately 70 seconds after the start of contrast medium administration, images were obtained at 2.5 and $5 \mathrm{~mm}$ thick axial slices starting from the superior of diaphragm to the level of the symphysis pubis while holding their breath. In order to create multi-planar reconstruction images, CT images were reconstituted with $1 \mathrm{~mm}$ thick pieces, at a reconstruction interval of $1 \mathrm{~mm}$. For reconstruction, thin-piece CT data were transferred to the 3D working station and coronal and sagittal MPR images were constituted with $3 \mathrm{~mm}$ intervals and $3 \mathrm{~mm}$ slice thickness.

\section{MRI}

Investigations were made with phased-array body coil as routine upper-abdomen MRI in Siemens Magnetom Symphony (Siemens, Erlangen, Germany) device. The field strength of the device is 1.5 Tesla $(\mathrm{T})$, which is considered to have a high field strength. Gradient force of superconductive (Niobium-Titanium) magnetic is $30 \mathrm{mT} / \mathrm{m}$ and FOV width is $400 \mathrm{~cm}$.

Before the diffusion weighted procedure, T2weighted True-FISP at axial plane (TR, 4.4s; TE, 2.2s; Average, 2; FlipAngle, 80ㅜ; Matrix, 256 x 256; number of sections, 25; thickness of sections, $5 \mathrm{~mm}$; FOV, 300; Average, 2; gap between sections, 15\%) sequence and after that diffusion-weighted, single-shot, echo-planar spin sequence, and chemical shift selective fat suppression technique (TR/ TE, 3700/76; Matrix, 128x128; number of sections, 30; FOV, 400; gap between sections, $15 \%$; section thickness, $5 \mathrm{~mm}$; duration, 156s; PAT Factor, 2; PAT mode, parallel imaging (GRAPPA) with modified sensitivity encoding) was applied. In DWI, b values were set as 0,400 and $800 \mathrm{~mm}^{2} /$ second.

\section{Evaluation of images}

An examination was conducted after installing diffusion-weighted images to the work station. All abdominal imaging MDCT and DWI results were assessed by two radiologists (HA, $\mathrm{AB}$ ) who had 12 and 10 years of experience, respectively. Two radiologists were informed about the localization of the lesions, which were diagnosed with

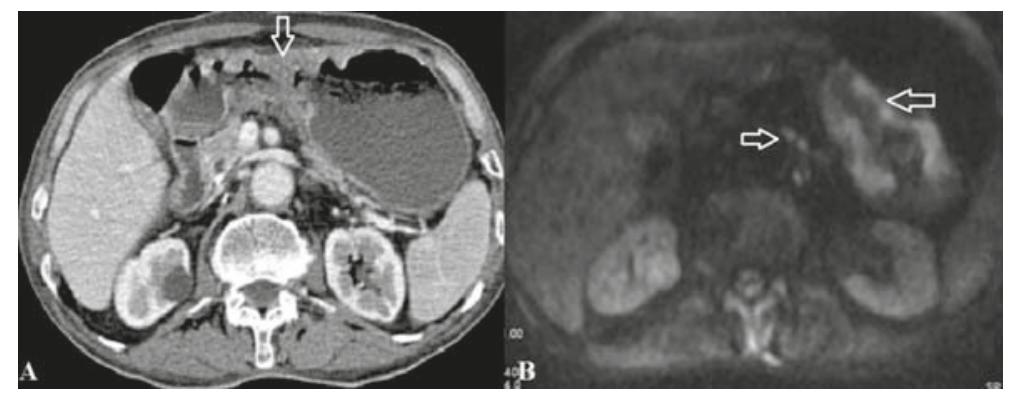

FIGURE 3. (A, B) T2N2MO Axial MDCT and b800 DWI. (A) Axialcontrast-enhanced MDCT and (B) b800 DWI demonstrate wall thickness in the gastric corpus withdiffusion restrictions, andadjacent lymphadenopathies ( $\mathrm{mm}$ sized) on DWI.

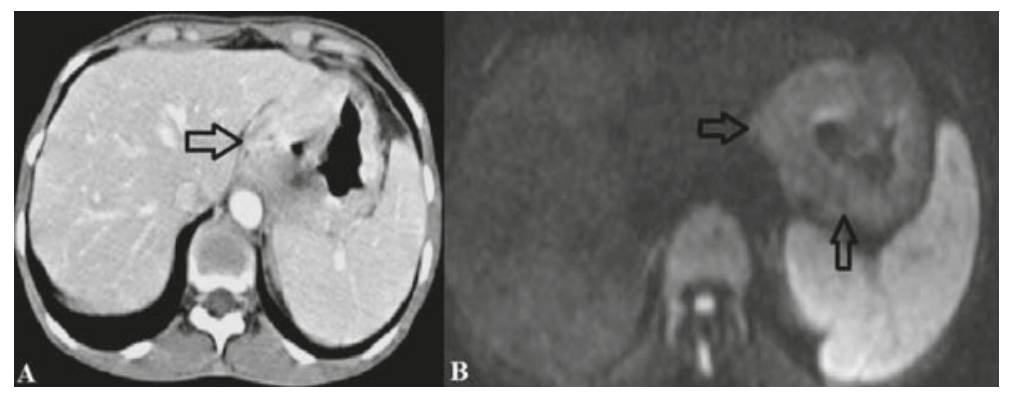

FIGURE 4. (A, B) T3NIMO Axial MDCT and DWI. (A) Axialcontrast-enhanced MDCT and (B) DWI demonstratewall thickness at gastric corpus and diffusion restriction and adjacent lymphadenopathieswere observed (arrow).

gastric cancer due to endoscopic biopsy. However, they were blinded to the pathological findings. MDCT and DWI image analysis were performed. Reviewers were told to determine $\mathrm{T}(\mathrm{T} 1, \mathrm{~T} 2, \mathrm{~T} 3, \mathrm{~T} 4)$ stage and $\mathrm{N}$ stage $(\mathrm{N} 1, \mathrm{~N} 2, \mathrm{~N} 3)$ by classifying as positive or negative and $\mathrm{M}$ stage by classifying as MO or M1. In abdominal images, only DWI images were evaluated for staging. Conventional MR images were not assessed in the staging.

\section{Surgical pathological analysis}

Surgery was performed within the following two weeks after cross sectional imaging. Curative or palliative gastrectomy and LN dissection were applied to 48 patients out of total 51 patients who were included in this study. Open-and-closed laparotomy was applied to three patients. For each case, pathological TNM staging was reported according to the Seventh AJCC Guideline. ${ }^{11}$

\section{Determination of the depth of tumor invasion (T staging)}

We applied imaging criteria that were based on previous studies about $\mathrm{T}$ staging of gastric can- 
TABLE 1. Effectiveness of DWI and MDCT in $T$ staging according to surgical pathology result

\begin{tabular}{ccccc}
\hline \multicolumn{2}{c}{ T2 } & & T3 & T4 \\
\hline \multirow{4}{*}{ DWI } & Sensitivity & $72.7 \%$ & $71.0 \%$ & $55.6 \%$ \\
& Specificity & $77.5 \%$ & $80.0 \%$ & $92.9 \%$ \\
& PPV & $47.1 \%$ & $84.6 \%$ & $62.5 \%$ \\
& NPV & $91.2 \%$ & $64.0 \%$ & $90.7 \%$ \\
& Kappa (p) & $0.419(0.002)$ & $0.488(<0.001)$ & $0.506(<0.001)$ \\
& Sensitivity & $63.6 \%$ & $74.2 \%$ & $66.7 \%$ \\
\multirow{4}{*}{ MDCT } & Specificity & $77.5 \%$ & $80.0 \%$ & $95.2 \%$ \\
& PPV & $43.8 \%$ & $85.2 \%$ & $75.0 \%$ \\
& NPV & $88.6 \%$ & $66.7 \%$ & $93.0 \%$ \\
& Kappa (p) & $0.353(0.009)$ & $0.523(<0.001)$ & $0.647(<0.001)$ \\
\hline
\end{tabular}

$\mathrm{CT}=$ Computed Tomography; $\mathrm{DWI}=$ Diffusion Weighted Imaging; NPV = Negative Predictive Value; PPV= Positive Predictive Value

cer and were modified according to the Seventh AJCC Guideline..$^{10,11}$ For each T stage, imaging criteria were as follows: $\leq \mathrm{T} 2$ stage ( $\leq$ muscularis propria invasion), it is an abnormal growing mass or wall thickness with/without ulceration, which has trans-mural involvement and irregular external border. The thin outer layer around the tumor and clear perigastric fatty tissue is preserved. T3 stage (penetrates subserosal connective tissue without involvement of the visceral peritoneum or adjacent structures), transmural tumor involves the entire stomach wall and 1) clear perigastric fat plane around the tumor is maintained, or 2) fine perigastric oil spicules that extend throughout small and large omentum and do not spread beyond the adjacent perigastric vessels. T4 stage (invasion of serosa (visceral peritoneum) or adjacent structures) is a transmural tumor with irregular borders and perigastric oil infiltration, which involves the entire stomach wall and shows invasion beyond adjacent perigastric vessels.

\section{Detection of regional lymph node involvement ( $\mathrm{N}$ staging)}

Positive lymph nodes can be detected according to their sizes in MDCT or MRDWI. If the shortest diameter of the largest regional lymph node is $\geq 8$ $\mathrm{mm}$, the patient should be considered as $\mathrm{N}$ positive; if it is $<8 \mathrm{~mm}$, the patient should be considered as negative. If there is at least one regional lymph node that has a shortest diameter of $\geq 8$ $\mathrm{mm}$ in DWI or there is any lymph node that shows higher signal intensity than muscle, the patient was classified as $\mathrm{N}$-positive. ${ }^{7}$

\section{Detection of distant metastasis ( $M$ staging)}

If suspicious lesions were seen in the MDCT or DWI about distant metastasis such as the liver, adrenal gland, distant lymph nodes, and peritoneum involvement, the patient was considered to be M1.

\section{Statistical analysis}

In gastric tumors, the comparison of DWI, MDCT, and surgical pathology was made using SPSS version 20 (SPSS Inc. Chicago,IL, USA) software. TNM results of two different imaging techniques (DWI and $\mathrm{CT}$ ) and TNM results of surgical pathology, which is accepted as the gold standard method, was compared and assessed using Pearson's chisquare (chi-square) or Fisher's exact test depending on the situation. Conformity between two different imaging techniques (DWI and CT) and surgical pathology was evaluated with the Kappa test. Additionally, sensitivity, specificity, positive predictive value, negative predictive value, and accuracy ratio of diagnostic tests were calculated. $\mathrm{P}$ values $<0.05$ were considered statistically significant.

\section{Results}

\section{Pathologic TNM staging of gastric cancer}

A total of 51 cases were included in the analysis. In the results of both surgical pathology and other two diagnostic tools (DWI and MD CT); there were no cases in the T1 stage and N0 stage. Because of this, $\mathrm{T}$ staging was made for $\mathrm{T} 2$ and higher and $\mathrm{N}$ staging was made for $\mathrm{N} 1$ and higher stages. $\mathrm{T}$ and $\mathrm{N}$ staging were confirmed in 51 patients to whom gastrectomy and LN dissection were applied. It was confirmed that in DWI 17 patients and in MDCT, 16 patients were stage T2. In DWI 26 patients and in MDCT 27 patients were stage T3. It was confirmed that in DWI seven patients and in MDCT seven patients were stage T4a and in MDCT one patient was stage $4 \mathrm{~b}$. In 51 patients, lymph node was positive. In two patients, peritoneum involvement was confirmed by radiologic evaluation and they were evaluated as M1. Other 49 patients were assessed as M0. 


\section{Diagnostic performance of $\mathrm{T}$ staging in gastric cancer}

T staging (T2, T3, T4) diagnosis of DWI was different from surgical pathology result ( $\mathrm{p}$ values were: $0.03,<0.001$ and 0.002 respectively). Similarly, $\mathrm{T}$ staging of MDCT was also different from the surgical pathology results ( $\mathrm{p}$ values: $0.014,<0.001,<$ 0.001 , respectively). When we looked for Kappa compatibility test for both diagnostic tools their compatibility with surgical pathology was generally weak (Table 1).

\section{Diagnostic performance of $\mathbf{N}$ staging in gastric cancer}

When we compared results of the DWI and surgical pathology in the staging of the lymph node; staging of DWI (N1, N2, N3) was different from the surgical pathology (for the three stages, $\mathrm{p}<$ 0.001). Similarly, a significant difference was found between MDCT results and surgical pathology results in N staging (p values; 0.003, 0.009, and 0.009, respectively). When we looked for Kappa compatibility test for $\mathrm{N}$ staging (Table 2), we detected that DWI is compatible with surgical pathology at a moderate-fine level. However, the compatibility of MDCT was weak.

According to surgical pathology result, distant metastasis (M1) was detected in total two cases. For M1, DWI and MDCT had full compliance with the gold standard (Kappa $=1.00 \mathrm{p}<0.001)$. Sensitivity and specificity of both imaging techniques for detecting distant metastasis was 100\%.

\section{Discussion}

MRI and CT play an important role in the diagnosis and preoperative staging of gastric cancer., Gastric cancer generally does not have any symptoms when it is at a stage at which a complete cure can be achieved. On the other hand, when clinically detected, it is generally at an advanced stage (local advanced or metastatic stage). ${ }^{12}$ Most of our cases were detected at an advanced stage. An accurate assessment of the depth of the tumor invasion into the stomach wall, local lymph node involvement, and metastasis presence or absence is very important for the selection of therapeutic strategy. In spite of the development of methods such as computed tomography (CT), positron emission tomography (PET), magnetic resonance (MR), ultrasound (US), gastroscopy, double-contrast barium
TABLE 2. Efficiency of DWI and MDCT in N staging according to surgical pathology result

\begin{tabular}{ccccc}
\hline & & N1 & N2 & N3 \\
\hline \multirow{4}{*}{ DWI } & Sensitivity & $75.0 \%$ & $79.3 \%$ & $60.0 \%$ \\
& Specificity & $84.6 \%$ & $77.3 \%$ & $97.6 \%$ \\
& PPV & $60.0 \%$ & $82.1 \%$ & $85.7 \%$ \\
& NPV & $91.7 \%$ & $73.9 \%$ & $90.9 \%$ \\
& Kappa (p) & $0.549(<0.001)$ & $0.563(<0.001)$ & $0.649(<0.001)$ \\
& Sensitivity & $66.7 \%$ & $69.0 \%$ & $50.0 \%$ \\
& Specificity & $82.1 \%$ & $68.2 \%$ & $90.2 \%$ \\
& PPV & $53.3 \%$ & $74.1 \%$ & $55.6 \%$ \\
& NPV & $88.9 \%$ & $62.5 \%$ & $88.1 \%$ \\
& Kappa (p) & $0.448(0.001)$ & $0.367(0.008)$ & $0.418(0.003)$ \\
\hline
\end{tabular}

$\mathrm{CT}=$ Computed Tomography; $\mathrm{DWI}=$ Diffusion Weighted Imaging; NPV = Negative Predictive Value; PPV= Positive Predictive Value

X-ray, and endoscopic ultrasound (EUS), there are still difficulties in the diagnosis and staging. ${ }^{13}$ Preoperative staging of gastric cancers has gained more importance with the recent developments in endoscopic treatment and also minimally invasive treatments of gastric cancers such as laparoscopic surgery. ${ }^{14}$ Recently, other MRI methods have been put into routine use, as well as conventional MR imaging. One of these methods is diffusion weighted MR imaging (DWI). Most of the studies about DWI were conducted with the Single Shot EPI (SSEPI) technique. An image is taken in a time unit of less than a second with the SSEPI technique and thus physiological movement is frozen and image artifacts are reduced. ${ }^{15}$ We used the SSEPI technique in the current study. Diffusion weighted MR imaging makes it possible to obtain information about the perfusion and diffusion of tissue at the same time, so normal and abnormal tissues can be distinguished from each other. Various abnormalities can be detected because of this technique. ${ }^{15}$ Today, the only imaging method that provides information about tumor cellularity is DWI. ${ }^{16}$ This cellular density difference reflects in the DWI. Various studies have reported that diffusion weighted MRI provides an important contribution to the diagnosis in the characterization of the tumor at different sites such as the liver, pancreas, ovary, colon, cervix, bladder, prostate, and breast. In association with cellularity of tumors, it was specified that malignant tumors have more diffusion limitations compared to benign lesions. ${ }^{17}$

So far, very few studies compared diagnostic performance of MDCT and 1.5-3 T MRDWI for 
the local staging of gastric cancer and showed that DWI is comparable with MDCT in T staging. ${ }^{7,8,18}$ Many studies have recently shown that using MDCT with MPR imaging in T staging is accurate with a ratio of $83 \%$ and $91 \%$, respectively. ${ }^{8,19}$

The current study demonstrated that DWI did not increase diagnostic performance significantly in $\mathrm{T}$ staging. In other studies that compared DWI and MDCT, DWI generally used for staging with conventional MRI and their accuracy values were high. ${ }^{8}$ In our study, using only DWI images were compared with MDCT in staging. We related low accuracy ratios in $\mathrm{T}$ staging with this condition. DWI and MDCT results in T staging can be better as the $\mathrm{T}$ stage increases compared to the gold standard. As T stage progresses, the effectiveness of DWI is related to an increase of size and density of tumor cells and limitation of intracellular diffusion during the impairment of regulation of tumor cells. As a result of these features, this study revealed that DWI could define advanced gastric cancer but it was not an effective method to detect early gastric cancers (T0-T1). We detected that it was possible to have similar results with almost the same accuracy with DWI for detecting wall invasion (T2, T3, T4) in 51 patients with gastric cancer when compared to MDCT.

Although the results of our work, in terms of $\mathrm{N}$ staging, did not have any significant difference for diagnostic accuracy between treatment methods, it was shown that DWI had significantly higher sensitivity for evaluating metastatic lymph nodes when compared with MDCT. Lymph node involvement is one of the most important prognostic factors, especially in gastric cancer. Lymph node metastasis is frequent in gastric cancer patients. ${ }^{20}$ However, as surgery related mortality and complications increase in the scope of analysis of the lymph nodes, it has been an issue that has been debated for a long time. In this context, for appropriate treatment strategies, local metastatic lymph nodes should be correctly considered before surgery. ${ }^{21,22}$ To this day, the diagnostic accuracy of MDCT in the N staging of gastric cancer varies between $46 \%$ and $83 \%$. 822 The weak and variable diagnostic performance might be a result of current lymph node metastasis criteria, which is generally based on lymph node size and shape; however, there can be microscopic metastasis in small sized lymph nodes and benign nodes usually can expand in gastric cancer patients when compared with a healthy population. It should be considered that lymph node size might not be adequate to distinguish metastatic lymph nodes from benign lymph nodes, especial- ly in gastric cancer patients. ${ }^{22,12}$ DWI also shows more diagnostic accuracy for detecting metastatic lymph nodes when compared to measurements in MRI and CT imaging. In addition to lymph node findings, there is potential to predict lymph node metastases with computerized analysis of tumor characteristics, such as primary tumor localization, diameter, Borrman types, histological types, infiltration depth, serosa invasion, molecular, and genetic markers. ${ }^{7}$ In the current study, apparent diffusion restriction is observed in lymph nodes with millimeter dimension due to diffusion weighted MRI and these were accepted as metastatic lymph nodes were confirmed after surgical pathology results (Figure 2). Malignant lesions are generally more cellular than benign lesions and this feature can provide information about the perfusion and diffusion of a tissue. Because of this high cellularity, malignant lesions have higher signal density in DWI and this demonstrates that DWI is more beneficial than MDCT to differentiate malignant lesions from benign ones. Moreover, the accuracy of $\mathrm{N}$ staging with DWI was statistically higher than the accuracy of N staging with MDCT. Furthermore, when combined with other morphological characteristics, DWI reached a higher predictive power than MDCT. In our study, more lymph nodes, especially under $1 \mathrm{~cm}$, were detected when evaluated with DWI. DWI signal changes could have higher predictive power than other morphological factors, including contrast uptake pattern and border irregularity for distinguishing metastatic and benign lymph nodes.

Distant metastases of gastric cancers include liver metastasis and peritoneum involvement. They can be more clearly detected by using DWI without any contrast agent when compared with MDCT. In our study, distant metastasis in two gastric cancer patients included peritoneum involvement and liver metastasis and these were detected with both DWI and MDCT. There was no statistically significant difference between these two methods. This might be due to the low number of metastatic patients in the study.

There were some limitations in this study. First of all, a relatively high proportion of patients in stage T3 might cause a statistical bias. Secondly, we did not detect any T1 stage cancer in the patient population. For this reason, further studies are required with more patients that include T1 stage cancer. Thirdly, as each lymph node was not compared separately for N staging, we could not match imaging and pathology diagnosis for each lymph node. Fourthly, as we used only qualitative 
assessment in DWI without using any quantitative measurement or size criteria, further studies are required to determine optimum diagnostic criteria with DWI in TNM staging of gastric cancer. Finally, two radiologists were informed about the localization of lesions but they were blinded to other endoscopic and surgical pathological findings.

In conclusion, DWI has a potential clinical use field, especially for the assessment of node involvement in gastric cancer. In particular, the conventional MRI and DWI combination might produce accurate performance with high sensitivity and specificity in lymph nodes smaller than one centimetre. When analyzed from a clinical viewpoint, an accurate lymph node metastasis evaluation before operation facilitates lymphadenectomy surgery and may reduce the risk of complications.

\section{References}

1. Shinya S, Sasaki T, Nakagawa Y, Guiquing Z, Yamamoto F, Yamashita Y. The usefulness of diffusion-weighted imaging (DWI) for the detection of gastric cancer. Hepatogastroenterol 2007; 54: 1378-81.

2. Dan YY, So JB, Yeoh KG. Endoscopic screening for gastric cancer. Clin Gastroenterol Hepatol 2006; 4: 709-16.

3. Smyth E, Schöder H, Strong VE, Capanu M, Kelsen DP, Coit DG, et al. A prospective evaluation of the utility of 2-deoxy-2-[(18) F]fluoro-D-glucose positron emission tomography and computed tomography in staging locallyadvanced gastric cancer. Cancer 2012; 118: 5481-88.

4. Kim JI, Kim YH, Lee KH, Kim SY, Lee YJ, Park YS, et al. Type-specific diagnosis and evaluationof longitudinal tumor extent of borrmann type IV gastric cancer: CT versus gastroscopy. Korean J Radiol 2013; 14: 597-606.

5. Makino T, Fujiwara Y, Takiguchi S, Tsuboyama T, Kim T, Nushijima Y, et al. Preoperative $\mathrm{T}$ stagingof gastric cancer by multi-detector row computed tomography. Surgery 2011; 149: 672-79.

6. Giganti F, De Cobelli F, Canevari C, Orsenigo E, Gallivanone F, Esposito A, et al. Response to chemotherapy in gastric adenocarcinoma with diffusionweighted MRI and F-FDGPET/CT: correlation of apparent diffusion coefficient and partial volume corrected standardized uptake value with histological tumorregression grade. J Magn Reson Imaging 2014; 40: 1147-57.

7. Liu S, He J, Guan W, Li Q, Zhang X, Mao H, et al. Preoperative T staging of gastric cancer: comparison of diffusion- and T2-weighted magnetic resonance imaging. J Comput Assist Tomogr 2014; 38: 544-50.

8. Joo I, Lee JM, Kim JH, Shin Cl, Han JK, Choi BI. Prospective comparison of 3T MRI with diffusion-weighted imaging and MDCT for thepreoperative TNM staging of gastric cancer. J Magn Reson Imaging 2015; 41: 814-21.

9. Bonekamp S, Corona-Villalobos CP, Kamel IR. Oncologic applications of diffusion-weighted MRI in the body. J Magn Reson Imaging 2012; 35: 257-79.

10. Avcu S, Arslan H, Unal O, Kotan C, Izmirli M. The role of diffusion-weighted $M R$ imaging and $A D C$ values in the diagnosisof gastric tumors. JBR-BTR 2012; 95: 1-5.

11. Edge SB BD, Compton CC, Fritz AG, Greene FL, Trotti A, editors. AJCC cancer staging manual. 7th edition. New York, NY: Springer; 2010.

12. Chen CY, Hsu JS, Wu DC, Kang WY, Hsieh JS, Jaw TS, et al. Gastric cancer: preoperative local staging with 3D multi-detector row CT--correlation with surgical and histopathologic results. Radiology 2007; 242: 472-82.

13. Lian J, Chen S, Zhang Y, Qiu F. A meta-analysis of endoscopic submucosal dissection and EMR for early gastric cancer. Gastrointest Endosc 2012;76: 763-70.
14. Schuhmacher C, Reim D, Novotny A. Neoadjuvant treatment forgastric cancer. J Gastric Cancer 2013; 13: 73-8.

15. Chow LC, Bammer R, Moseley ME, Sommer FG. Single breath-hold diffusionweighted imaging of the abdomen. J Magn Reson Imaging 2003; 18: $377-82$.

16. Naganawa S, Sato $C$, Kumada H, Ishigaki T, Miura S, Takizawa O. Apparent diffusion coefficient in cervical cancer of the uterus: comparison with the normal uterine cervix. Eur Radiol 2005; 15: 71-8.

17. Kuroki Y, Nasu K, Kuroki S, Murakami K, Hayashi T, Sekiguchi R, et al. Diffusion-weighted imaging of breast cancer with the sensitivity encoding technique: analysis of the apparent diffusion coefficient value. Magn Reson Med Sci 2004; 3: 79-85.

18. Shen L, Huang Y, Sun M, Xu H, Wei W, Wu W. Clinicopathological features associated with lymph node metastasis in early gastric cancer: analysis of a single-institution experience in China. Can J Gastroenterol 2009; 23: 353-6.

19. Kumano S, Okada M, Shimono T, Kuwabara M, Yagyu Y, Imaoka I, et al. T-staging of gastric cancer of air-filling multidetector-row CT: comparison with hydromultidetector-row CT. Eur J Radiol 2012; 81: 2953-60.

20. Cheng J, Wang Y, Deng J, McCarthy RJ, Wang G, Wang H, et al. Discrimination of Metastatic Lymph Nodes in Patients with Gastric Carcinoma UsingDiffusion-Weighted Imaging. J Magn Reson Imaging 2013; 37: 143644.

21. Kwee RM, Kwee TC. Imaging in assessing lymph node status ingastric cancer. Gastric Cancer 2009; 12: 6-22.

22. Park HS, Kim YJ, Ko SY, Yoo MW, Lee KY, Jung SI, et al. Benign regional lymph nodes in gastric cancer on multidetector row CT. Acta Radiol 2012; 53: 501-7. 